\section{Scattering of Hard $\gamma$-Rays and Annihilation Radiation}

To determine the relative importance of the annihilation of positive electrons in the phenomenon of the 'scattering' of hard $\gamma$-rays, observations were made on the secondary $\gamma$-radiation from thin lead foils irradiated with thorium $\mathrm{C}^{\prime \prime} \gamma$-rays $(25 \mathrm{mgm}$.). With a scattering angle of about $140^{\circ}, 0.8 \mathrm{~cm}$. effective lead filter was sufficient to absorb practically all the Compton scattering, and still leave predominant the softer of the two components in the anomalous scattering, namely, the component which has been attributed to the annihilation of positive electrons.

A sheet of paraffin wax was placed in front of the source to reduce to a minimum the number of positive electrons falling on the scatterer. Measurements were made on the secondary $\gamma$-radiation from foils of varying thicknesses $(t)$ down to thicknesses of the order of, and appreciably less than, the average range $(R)$ of the positive electrons, expected from theory, from the $\gamma$-rays used. If the annihilation hypothesis is correct, the 'scattered' radiation under these conditions should, in the region $t \sim R$, decrease much more rapidly with decreasing $t$ than its first power, owing to the escape of the positive electrons from the foil before annihilation. This rapid decrease was actually found, the effect of the thinnest foil used $(\sim 0.002 \mathrm{~cm}$.$) being only about 30$ per cent of the value corresponding to a linear variation with $t$. This shows that at least about 70 per cent of the scattered radiation under these conditions is due to annihilation ${ }^{1}$.

To test further the annihilation theory of the origin of the soft component, it was compared, under practically identical conditions, with the Compton scattering through $79^{\circ}$, this angle of scattering giving Compton radiation of the same wave-length, namely $h / m c$, as that of the theoretical annihilation radiation. Both in frequency and intensity the soft component was found to agree fairly well with the theoretical expectations, and there is no question, according to the present experiments, of the annihilation radiation being only a small fraction of the theoretical value, as maintained by Bothe and Horn ${ }^{2}$. The theoretical intensity was estimated in the usual way from the number of positive electrons produced by the primary $\gamma$-rays according to Dirac's theory ${ }^{3}$.

To investigate the hard component in the anomalous scattering, observations were made with different thicknesses of lead filter in the path of the secondary rays, up to $5 \mathrm{~cm}$. of lead at $140^{\circ}$, and up to $7 \mathrm{~cm}$. at $80^{\circ}$. While a slight progressive hardening was found with increasing filter strength, throughout this range, there was no evidence for secondary radiation of primary hardness. Actually, with the maximum filter thickness, the secondary radiation was definitely softer than the hard $\gamma$-rays from radium $C$. The latter correspond to a quantum energy of about $1.9 \times 10^{6}$ volts, while the effective primary radiation in the above experiments has a quantum energy of $2.65 \times 10^{6}$ volts.

The initial intensity of the hard component from lead is of the order of 15 per cent of that of the half-million volt radiation. It is somewhat anisotropic, being more intense at $80^{\circ}$ than at $140^{\circ}$. The anisotropy is more pronounced for light elements. These results are qualitatively in agreement with the supposition that the hard component is due to X-radiation from Compton electrons, and the annihilation of positive electrons before reaching the end of their range, the degree of anisotropy of such tertiary radiations being dependent on the atomic number through the nuclear scattering of the secondary electrons. The importance of the first of these processes has already been emphasised by Lauritsen and Oppenheimer ${ }^{4}$, and from more recent and detailed calculations by Wheeler and Plesset ${ }^{5}$ it appears that the two processes will account for at least a large part of the hard component.

A fuller account of the experiments described in this note, and of a more detailed analysis of the results when completed, will be published in due course. The experiments were carried out at the laboratory of the Institute for Theoretical Physics, Copenhagen, and I should like to take this opportunity of thanking Prof. N. Bohr for his kind interest in the investigation, and also Dr. J. C. Jacobsen and Mr. K. F. Brostrøm for their valuable assistance in the experimental work.

Institute for Theoretical Physics,

\section{E. J. WIILTAMS} Copenhagen. Jan. 12.

${ }^{1}$ Cf. earlier experiments by the writer using $0.1 \mathrm{~mm}$. lead foil and radium C $\gamma$-rays (NATURE, 133, 415; 1934).

2 $Z$. Phys., 88, $683 ; 1934$.

s Oppenheimer and Plesset, Phys. Rev., 44, 53; 1933. Bethe and Heitler, Proc. Roy. Soc., 148, $83 ; 1934$.

Phys. Rev., 48, 80 ; 1934

5 To be published in the Physical Review. These authors have also considered other possible effects, including the Raman scattering of the primary radiation, and found them to be unimportant.

\section{Colchicine and Tumour Growth}

THE finding of considerable numbers of mitotic figures in the hæmopoietic organs of normal healthy animals and in the neoplastic tissues of tumourbearing animals after colchicine administration has led many students to suspect an inter-relationship between mitosis and the alkaloid. But the effect of colchicine in slowing down the rate of growth of neoplastic tissue has not been reported.

Following on some earlier observations (unpublished, 1927) which I made in conjunction with the late Prof. M. R. J. Hayes on the beneficial effects of deep X-ray therapy on neoplasms in patients suffering from acute attacks of gout, which were being treated with colchicum, a series of experiments was recently planned with the object of determining to what extent the colchicine might affect new growths.

In one of these, a group of twelve tumour-bearing mice $(M 63)$, were injected subcutaneously on alternate days with small doses of colchicine (kindly supplied by the director of the Wellcome Bureau of Scientific Research, where the work was carried out). Treatment lasted for a period of two weeks. Twelve other tumour-bearing mice were used as controls. The tumours at the end of the first week showed much less growth as compared with the controls; while at the end of the second week there was no macroscopically recognisable tumour tissue present in $66 \cdot 6$ per cent of the injected animals, and only minute nodules could be detected in the remaining $33 \cdot 3$ per cent. These nodules finally showed complete regression, and no tumour tissue could be recognised eight weeks later. The controls in all but one instance showed a marked development of the tumour. In another series, the percentage of animals in which there was no recognisable tumour tissue at the end of two weeks was 100 per cent.

From the effects observed in these tumour-bearing mice, it was thought advisable to determine what 